\title{
Formulation and Characterization of Topical Anti-acne Spot Gel Containing Herbal Extracts
}

\author{
Jirapornchai Suksaeree ${ }^{1, a}$ and Chomnapas Chuchote Ch $^{2, b}$ \\ ${ }^{1}$ Department of Pharmaceutical Chemistry, College of Pharmacy, Rangsit University, Pathum Thani, Thailand \\ ${ }^{2}$ Department of Pharmacognosy, College of Pharmacy, Rangsit University, Pathum Thani, Thailand
}

\begin{abstract}
This research work aimed to prepare the topical anti-acne spot gel incorporating the crude polyherbal extract that is a traditional Thai herbal recipe, namely "Ya-Sa-Marn-Phlae". The topical anti-acne spot gels were prepared by add the crude polyherbal extract in each microemulsion or self-microemulsifying system and subsequently mixed in the blank anti-acne spot gel. The $\mathrm{pH}$, viscosity, stability, contamination test, and skin irritation test were evaluated. The topical anti-acne spot gel formulations had a yellowish in color that was visually checked by researcher and photographed by digital camera. They had a $\mathrm{pH}$ near to a human skin and good viscosity which ease to use and safe to contact to the skin. They had a good stability and no contamination of Staphylococcus aureus and Escherichia coli. The topical anti-acne spot gels exhibited no oedema formation, and very slight erythema formation. Thus, the topical antiacne spot gels were safe to apply on the skin without irritation.
\end{abstract}

\section{Introduction}

In the recent years, herbal formulations from Thai herbal compounds are the good demand resulting in its rapid growth in Thailand. They are more acceptable and interesting in the view that they are less side effects and safe than the synthetic products. The herbal products are continuously developed and has been reported by incorporated the Thai herbal compounds such as curcumin [1], quercetin [2], paclitaxel [3], tea catechins [4], capsaicin [5, 6], Zingiber cassumunar [7, 8] etc. The main objectives of herbal products are delivery of the active compound with constant release rate over the period of treatment and channel for entity of the active compound to the site of action $[9,10]$.

This present work used the traditional Thai herbal recipe, namely "Ya-Sa-Marn-Phlae", to formulate the topical anti-acne spot gel formulation. This traditional Thai herbal recipe composed of Curcuma longa L., Areca catechu L., Oryza sativa L., and Garcinia mangostana L. in ratio of 1:1:1:1, respectively found as a treatment of wounds and skin infections that described in elsewhere [11, 12]. The crude polyherbal extract is prepared and tested by Dr.Chonlatid Sontimuang, Faculty of Traditional Thai Medicine, Prince of Songkla University.

The objective of this study was the preparation of the topical anti-acne spot gel containing the polyherbal extract. The polyherbal extract was entrapped in each microemulsion (ME) or selfmicroemulsifying (SME) system before mixing in the topical anti-acne spot gel. However, the suitable

\footnotetext{
${ }^{a}$ Corresponding author : jirapornchai.s@rsu.ac.th

${ }^{\mathrm{b}}$ Corresponding author : chomnapas@gmail.com
} 
ME and SME systems are observed and optimized via our group which reported in elsewhere [13, 14]. The only one of the optimized system for each ME and SME was selected to prepare the topical antiacne spot gel. The topical anti-acne spot gels were determined and evaluated the $\mathrm{pH}$, viscosity, stability, contamination test, and skin irritation test.

\section{Experimental Works}

\subsection{Raw materials and chemicals}

Caprylic acid, Tween ${ }^{\circledR} 20$, Tween ${ }^{\circledR} 80$, propylene glycol, carbomer, Triethanolamine, etc. were purchased from P.C. Drug Center Co., Ltd, Thailand. The polyherbal extract is prepared by Dr.Chonlatid Sontimuang. Briefly, the polyherbal formulation composed of C. longa L., A. catechu L., O. sativa L., and G. mangostana L. in ratio of 1:1:1:1, respectively. The mixture herb was extracted in $95 \%$ ethanol and water at room temperature for 7 days. Then, the solution of polyherbal extract was evaporated the ethanol by rotary evaporator and freeze-dried to remove the water. Finally, the crude polyherbal extract was sterilized at $-20^{\circ} \mathrm{C}$.

\subsection{Preparation of ME and SME}

The composition of the ME and SME base showed a clear and thermodynamically stable system are chosen according to preliminary trials explained of the pseudoternary phase diagrams in a previous work $[13,14]$. ME system composted of the $20 \% \mathrm{w} / \mathrm{w}$ of caprylic acid as an oil phase, $70 \% \mathrm{w} / \mathrm{w}$ of mixture of Tween ${ }^{\circledR} 80$ and absolute ethanol $(8: 2)$ as a surfactant, and $10 \% \mathrm{w} / \mathrm{w}$ of water phase. SME system composed of $10 \% \mathrm{w} / \mathrm{w}$ of caprylic acid as an oil phase, $60 \% \mathrm{w} / \mathrm{w}$ of mixture of Tween ${ }^{\mathbb{B}} 80$ and absolute ethanol $(8: 2)$ as a surfactant, and $30 \% \mathrm{w} / \mathrm{w}$ of propylene glycol as a co-surfactant phase without water phase. Then, the $1 \% \mathrm{w} / \mathrm{w}$ Thai herbal extract was dissolved in optimized ME or SME system. The systems were prepared by vortex-mixed for 30 second. The monophasic formulations were formed spontaneously at room temperature.

\subsection{Preparation of topical anti-acne spot gel formulation containing polyherbal extract}

The clear and thermodynamically stable system that had a good clear emulsion in appearance was mixed in base formulation of topical anti-acne spot gel that are presented in Table 1. All gradients were easily mixed in beaker.

Table 1. The composition of blank and topical anti-acne spot gel formulations

\begin{tabular}{cccccccc}
\hline Formulas & $\begin{array}{c}\text { Polyherbal } \\
\text { extract } \\
(\% \mathrm{w} / \mathrm{w})\end{array}$ & $\begin{array}{c}\mathrm{ME} \\
(\% \mathrm{w} / \mathrm{w})\end{array}$ & $\begin{array}{c}\mathrm{SME} \\
(\% \mathrm{w} / \mathrm{w})\end{array}$ & $\begin{array}{c}\text { Carbomer } \\
(\% \mathrm{w} / \mathrm{w})\end{array}$ & $\begin{array}{c}\text { Propylene } \\
\text { glycol } \\
(\% \mathrm{w} / \mathrm{w})\end{array}$ & $\begin{array}{c}\text { Paraben } \\
\text { concentration } \\
(\% \mathrm{w} / \mathrm{w})\end{array}$ & $\begin{array}{c}\text { Triethanolamine } \\
(\% \mathrm{w} / \mathrm{w})\end{array}$ \\
\hline B1 & - & 10 & - & 0.9 & 20 & 1 & 0.45 \\
B2 & - & - & 10 & 0.9 & 20 & 1 & 0.45 \\
F1 & 1 & 10 & - & 0.9 & 20 & 1 & 0.45 \\
F2 & 1 & - & 10 & 0.9 & 20 & 1 & 0.45 \\
\hline
\end{tabular}

\subsection{Characterization}

The Nanoplus 3 (Particulate system, USA) was used to determine the particle size, size distribution, and zeta potential at $25 \pm 2^{\circ} \mathrm{C}$. The $\mathrm{pH}$ and viscosity of topical anti-acne spot gel containing polyherbal extract was measured by a SevenEasy S-20 pH meter (Mettler Toledo, Switzerland) and a Brookfield viscometer (Brookfield engineering laboratories Inc, USA) at $25 \pm 2{ }^{\circ} \mathrm{C}$ with the S-96 spindle at 100 
rpm, respectively. Each topical anti-acne spot gel containing polyherbal extract was measured in triplicate. The

\subsection{Stability test}

The formulation was kept at $40 \pm 2^{\circ} \mathrm{C}$ and $75 \% \mathrm{RH}$ for $0,1,2$, and 3 months (Method I). Freeze - thaw stability test, the formulation was kept in refrigerator at $4 \pm 2^{\circ} \mathrm{C}$ for 24 hours and subsequently moved to $40 \pm 2^{\circ} \mathrm{C}$ for 24 hours and repeated all step for 6 cycles (Method II).

\subsection{Contamination test}

The formulation was diluted to $10^{-1}, 10^{-2}$, and $10^{-3} \mu \mathrm{g} / \mathrm{mL}$ of concentration with $0.9 \%$ sodium chloride solution, and then speeded onto Petri-dish that contained each manital salt agar (Staphylococcus aureus) or MacConkey agar (Escherichia coli) contamination test. They were incubated at $37 \pm 2^{\circ} \mathrm{C}$ for 24 hours.

\subsection{Skin irritation test}

The topical anti-acne spot gel formulation containing polyherbal extract was applied onto the skin of healthy adult albino rabbits; the New Zealand white hybrid strain obtained from the Department of Animal Science, Faculty of Agriculture, Kasetsart University, Thailand. It was evaluated by Thailand Institute of Scientific and Technology Research following the OECD guidelines for testing of chemicals (TG 404) with the Animal Ethics Committee approval number TS-59001 [15]. The rabbit's skin was assessed on each site at 1, 24, 48, and 72 hours after removal of the patches. The skin irritation was graded according to the scale originally proposed by Draize test skin.

\section{Results and discussion}

The crude polyherbal extract is prepared and tested by Dr.Chonlatid Sontimuang. The crude polyherbal extract is the traditional Thai herbal recipe, namely "Ya-Sa-Marn-Phlae" is found that the antibacterial, anti-inflammatory, and antioxidant activities and low toxicity that described in elsewhere $[11,12]$ that will be used to develop a topical formula based on traditional knowledge. The Ya-Sa-Marn-Phlae recipe composed of the 4 Thai herbs that are C. longa L., A. catechu L., O. sativa $L$., and $G$. mangostana $L$. in ratio of $1: 1: 1: 1$, respectively.

From the previous work reported the preparation of the pseudoternary phase diagrams for ME and SME system that showed a clear and thermodynamically stable system [13, 14]. The best systems were $20 \% \mathrm{w} / \mathrm{w}$ of caprylic acid as an oil phase, $70 \% \mathrm{w} / \mathrm{w}$ of mixture of Tween ${ }^{\mathbb{B}} 80$ and absolute ethanol (8:2) as a surfactant, and $1020 \% \mathrm{w} / \mathrm{w}$ of water phase for ME system, and $10 \% \mathrm{w} / \mathrm{w}$ of caprylic acid as an oil phase, $60 \% \mathrm{w} / \mathrm{w}$ of mixture of Tween ${ }^{\mathbb{}} 80$ and absolute ethanol (8:2) as a surfactant, and $30 \% \mathrm{w} / \mathrm{w}$ of propylene glycol as a co-surfactant phase without water phase for SME system. The crude polyherbal extract was added in concentration of $1 \% \mathrm{w} / \mathrm{w}$ that showed a clear and thermodynamically stable system. The zeta potential, droplet size, and size distribution of optimized ME and SME system were determined that are shown in Table 2. Both ME and SME showed that the good stability and the droplet size of the dispersed phase was less than $200 \mathrm{~nm}$ with narrow distribution. The transparency of the both systems was observed which would not show aggregation effect due to charge of the droplets [16]. 
Table 2. The zeta potential, droplet size, and size distribution of optimized ME and SME system

\begin{tabular}{cccc}
\hline Formulas & Zeta potential $(\mathrm{mV})$ & Droplet size $(\mathrm{nm})$ & PI \\
\hline ME base & $-7.19 \pm 1.23$ & $192.30 \pm 1.97$ & 0.22 \\
Crude polyherbal extract - loaded ME & $-11.26 \pm 2.42$ & $177.00 \pm 0.26$ & 0.21 \\
SME base & $-8.22 \pm 0.59$ & $148.47 \pm 0.93$ & 0.30 \\
Crude polyherbal extract - loaded SME & $-7.89 \pm 0.65$ & $133.03 \pm 1.70$ & 0.23 \\
\hline
\end{tabular}

Figure 1 shows the appearance of blank topical anti-acne spot gels (left) and topical anti-acne spot gels (right). The appearance of the blank topical anti-acne spot gels was a white in color while the intensity of its color increased with the addition the polyherbal extract, which was a yellowish in color.

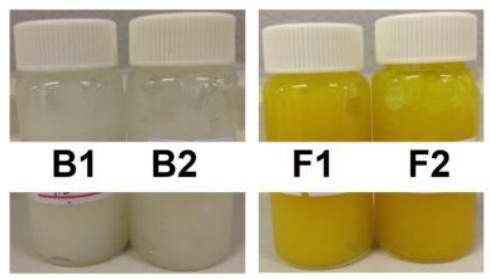

Figure 1. The appearance of blank topical gels (left) and topical anti-acne spot gels (right)

The $\mathrm{pH}$ and viscosity value of topical anti-acne spot gels are shown in Table 3. After crude polyherbal extract was added, it was found that not changed from those blank gel formulations. Thus, the crude polyherbal extract not affected on these values. The $\mathrm{pH}$ value of fresh preparation of topical anti-acne spot gel formulations were $6.01 \pm 0.01$ and $5.85 \pm 0.02$ for $\mathrm{F} 1$ and $\mathrm{F} 2$ formula, while the $\mathrm{pH}$ values were found that varied from $5.60-5.80$ after storage at $40 \pm 2^{\circ} \mathrm{C}$ for 3 months, and 5.79 to 5.66 after freeze - thaw test. However, this $\mathrm{pH}$ value was safe to use for skin applications that human skin surface $\mathrm{pH}$ is on average between $5.00-6.00$. Similarly, the viscosity after stability test of topical anti-acne spot gels slightly decreased, when compared to those fresh topical anti-acne spot gels. This might be the temperature effects which slightly decreased the movement of polymer chain. The appearance of topical anti-acne spot gel formulation after stability testing is shown in Figure 2. It was found that the intensity of its color was clearly observed to dark yellow after stability test. Results showed that the topical anti-acne spot gels stood stable at stability testing condition, both kept at $40 \pm 2^{\circ} \mathrm{C}$ with $75 \% \mathrm{RH}$ in stability chamber for 3 months and freeze - thaw test for 6 cycles, with a little change.

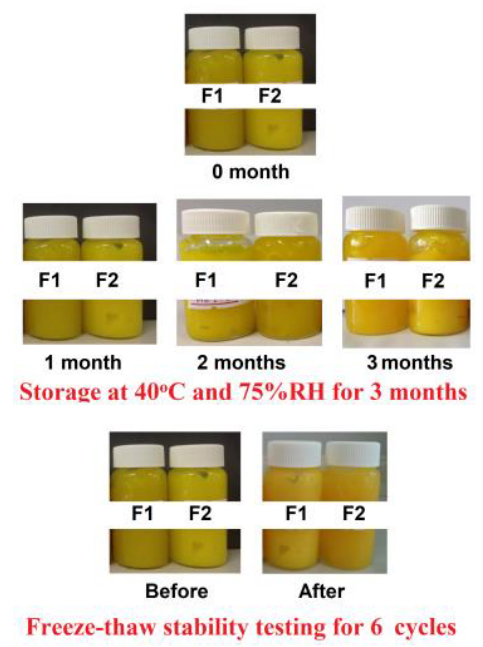

Figure 2 The appearance of topical anti-acne spot gel formulations after stability testing 
Table 3. $\mathrm{pH}$ and viscosity of blank and topical anti-acne spot gel formulations

\begin{tabular}{cccccc}
\hline \multicolumn{7}{c}{$\mathrm{pH}$ value } \\
\hline Formulas & 0 month & 1 month & 2 months & 3 months & Freeze - thaw stability \\
\hline B1 & $6.03 \pm 0.02$ & $6.00 \pm 0.01$ & $6.01 \pm 0.01$ & $5.93 \pm 0.02$ & $5.90 \pm 0.04$ \\
B2 & $5.90 \pm 0.02$ & $5.82 \pm 0.03$ & $5.80 \pm 0.02$ & $5.77 \pm 0.03$ & $5.70 \pm 0.03$ \\
F1 & $6.01 \pm 0.01$ & $5.88 \pm 0.01$ & $5.81 \pm 0.01$ & $5.80 \pm 0.02$ & $5.79 \pm 0.02$ \\
F2 & $5.85 \pm 0.02$ & $5.72 \pm 0.02$ & $5.64 \pm 0.01$ & $5.60 \pm 0.02$ & $5.66 \pm 0.04$ \\
\hline \multicolumn{7}{c}{ Viscosity value $\left(\times 10^{3} \mathrm{cps}\right)$} \\
\hline B1 & $340.89 \pm 30.71$ & $306.98 \pm 20.01$ & $288.96 \pm 20.82$ & $228.82 \pm 26.80$ & $338.10 \pm 31.82$ \\
B2 & $366.88 \pm 25.07$ & $340.70 \pm 29.02$ & $300.19 \pm 20.77$ & $280.72 \pm 28.91$ & $256.66 \pm 26.93$ \\
F1 & $334.33 \pm 25.90$ & $297.59 \pm 18.00$ & $245.39 \pm 21.08$ & $221.20 \pm 22.49$ & $302.75 \pm 24.30$ \\
F2 & $347.36 \pm 22.09$ & $322.15 \pm 21.12$ & $304.15 \pm 28.88$ & $269.47 \pm 26.54$ & $248.38 \pm 22.67$ \\
\hline
\end{tabular}

The contamination test of topical anti-acne spot gels was observed by using Manital Salt agar and MacConkey agar for $S$. Aureus and E. coli testing, respectively at $37 \pm 2^{\circ} \mathrm{C}$ for 24 hours which presents in Figure 3. The contamination was not found in topical anti-acne spot gels at different diluted concentration

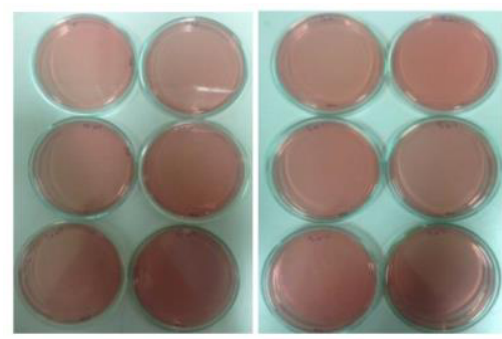

Manital Salt agar

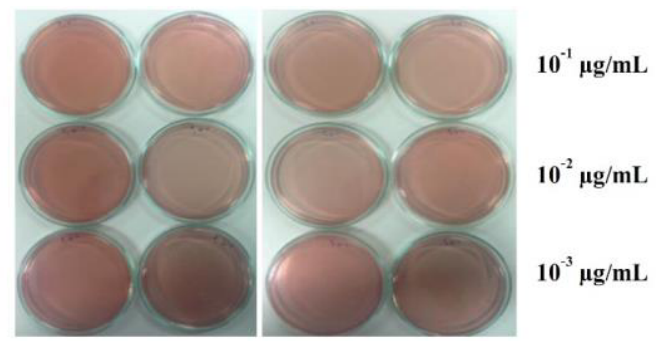

MacConkey agar

Figure 3 The antimicrobial sensitivity testing for S. aureus (Manital Salt agar) and E. Coli (MacConkey agar)

The topical anti-acne spot gels were further tested in vivo by acute dermal irritation test in New Zealand white rabbits. The scores of dermal reactions: erythema and eschar formation, and oedema formation, were used as an index of skin damage caused by these topical anti-acne spot gels. When the distilled water was treated as a control area on rabbit's test, it was found that no sign of any erythema and eschar formation, and oedema formation. Unlucky, when the topical anti-acne spot gels were treated on tested rabbit's skin, one of all tested rabbits exhibited a very slight erythema formation ( score $=1$ ) on the tested skin at 1 hour after removal of the gels and any residual, and this effect not exhibited at 24 hours, while the other rabbit showed no erythema (scale 0 ) at any time of the investigations. After that, they were not found the erythema and eschar formation until 72 hours. All tested rabbits showed that no oedema formation ( score $=0$ ). The effect of high content of surfactant was used to describe for this damage on the rabbit's skin. The degree of skin irritation potential of test substances that had various number of factors such as species of animals used. The results of skin irritation may be changed if the different animal models have been employed. Generally, the laboratory animals are more susceptible to chemicals than humans [17]. However, the skin irritation on human skin testing will be tested to provide practical information on potential skin damages of these topical anti-acne spot gels.

\section{Conclusions}

The topical anti-acne spot gels were initially prepared by our research group by add the crude polyherbal extract in each ME or SME system and subsequently mixed in blank anti-acne spot gel. 
The topical anti-acne spot gels had a yellowish in color with good stability and no contamination. The $\mathrm{pH}$ and viscosity were found that safe to use on the skin. The topical anti-acne spot gels exhibited no oedema formation, and very slight erythema formation at 1 hour and was not presented until 72 hours with no eschar formation. Thus, the topical anti-acne spot gel formulations were safe to apply on the skin without irritation.

\section{Acknowledgements}

The authors wish to thank KI Tull for English language assistance and Dr.Chonlatid Sontimuang, Faculty of Traditional Thai Medicine, Prince of Songkla University for prepared the polyherbal extract. This work was supported by the Faculty of Pharmacy and the Research Institute of Rangsit University.

\section{References}

1. K. Maiti, K. Mukherjee, A. Gantait, B.P. Saha, P.K. Mukherjee, Int. J. Pharm. 330, 155-63 (2007)

2. A. Priprem, J. Watanatorn, S. Sutthiparinyanont, W. Phachonpai, S. Muchimapura, Nanomed. Nanotechnol. Biol. Med. 4, 70-8

3. S. Rane, B. Prabhakar, Indian J. Pharm. Sci. . 75, 420-6 (2013)

4. J.-Y. Fang, T.-L. Hwang, Y.-L. Huang, C.-L. Fang, Int. J. Pharm. 310, 131-8 (2006)

5. P.R. Desai, S. Marepally, A.R. Patel, C. Voshavar, A. Chaudhuri, M. Singh, J. Controlled Release. 170, 51-63 (2013)

6. Y. Zhu, W. Peng, J. Zhang, M. Wang, C.K. Firempong, C. Feng, et al., J. Funct. Foods. 8, 358-66 (2014)

7. J. Suksaeree, L. Charoenchai, F. Madaka, C. Monton, A. Sakunpak, T. Charoonratana, et al., Asian J. Pharm. Sci. 10, 341-9 (2015)

8. J. Suksaeree, C. Monton, F. Madaka, T. Chusut, W. Saingam, W. Pichayakorn, et al., AAPS PharmSciTech. 16, 171-81 (2015)

9. S. Parasuraman, G.S. Thing, S.A. Dhanaraj, Pharmacogn. Rev. 8, 73-80 (2014)

10. Ajazuddin, S. Saraf, Fitoterapia. 81, 680-9 (2010)

11. S. Chusri, S. Tongrod, J. Chokpaisarn, S. Limsuwan, S.P. Voravuthikunchai, BioMed Research International. 2014, 6 (2014)

12. S. Chusri, S. Settharaksa, J. Chokpaisarn, S. Limsuwan, S.P. Voravuthikunchai, J. Altern. Complement. Med. 19, 671-6 (2013)

13. P. Maneewattanapinyo, K. Kantapak, P. Sansiri, N. Chatpitukpong, C. Sontimuang, J. Suksaeree, Thai J. Pharm. Sci. 40, 53-6 (2016)

14. P. Maneewattanapinyo, K. Kantapak, P. Sansiri, N. Chatpitukpong, C. Sontimuang, J. Suksaeree, Thai J. Pharm. Sci. 40, 57-60 (2016)

15. Organization for Economic Co-operation and Development (OECD), OECD Guidelines for Testing of Chemicals, Section 4: Health Effects, Test No. 404: Acute Dermal Irritation/Corrosion . (Paris Cedex 162002)

16. S.-x. Cui, S.-f. Nie, L. Li, C.-g. Wang, W.-s. Pan, J.-p. Sun, Drug Dev. Ind. Pharm. 35, 603-11 (2009)

17. S. Songkro, W. Pichayakorn, S. Sungkarak, J. Wungsintaweekul, J. drug deliv. sci. tech. 21, 40110 (2011) 